\title{
Was sich mit supportiver Therapie und neuen Ansätzen erreichen lässt
}

\begin{abstract}
Eigentlich genügt schon ein Blick ins Gesicht der Patienten, um zu erahnen, woran sie leiden, erklärte Prof. Dr. med. Wolf-Karsten Hofmann, Mannheim. Patienten mit myelodyspastischem Syndrom sind blass und kränkelnd und klagen über Schwäche und Luftnot. Therapeutisch wurden in den letzten Jahren deutliche Fortschritte erzielt. Der große Durchbruch steht aber noch aus.
\end{abstract}

— Die therapeutischen Möglichkeiten beim myelodysplastischen Syndrom (MDS) reichen von der Eisenchelation über hämopoetische Wachstumsfaktoren, immunmodulierende und demethylierende Substanzen bis hin zu experimentellen Ansätzen.

\section{Unterstützende Therapie}

Eine Chance auf Heilung haben allerdings nur die wenigsten Patienten. Große Bedeutung hat deshalb die supportive Therapie. Die Gabe von Erythrozytenkonzentraten (EK) orientiert sich nicht am Hb-Wert, sondern am klinischen $\mathrm{Zu}$ stand des Patienten. Die Transfusion von Thrombozyten beschränkt sich auf die Indikation einer erhöhten Blutungsneigung und sollte niemals prophylaktisch erfolgen, so Hofmann. Hingegen plädierte er für die frühzeitige Antibiotikagabe bei Infektionen und unterstrich die Notwendigkeit, andere internistische Krankheiten wie Herzinsuffizienz oder COPD zu behandeln.

\section{Polytransfundierte Patienten benötigen Eisenchelattherapie}

„Bei einem Großteil der Patienten mit einem MDS kommen Sie an einer regelmäßigen Transfusion nicht vorbei“, erklärte Hofmann. Nachdem die Patienten heute länger überleben als noch vor Jahren, kann es zu einer Eisenüberladung kommen (sekundäre Hämatochromatose). Bei einem polytransfundierten $\mathrm{Pa}$ tienten mit bis 52 EKs pro Jahr akkumuliert sich die Menge Eisen unter Umständen auf $13 \mathrm{~g}$ bei einem Verlust von

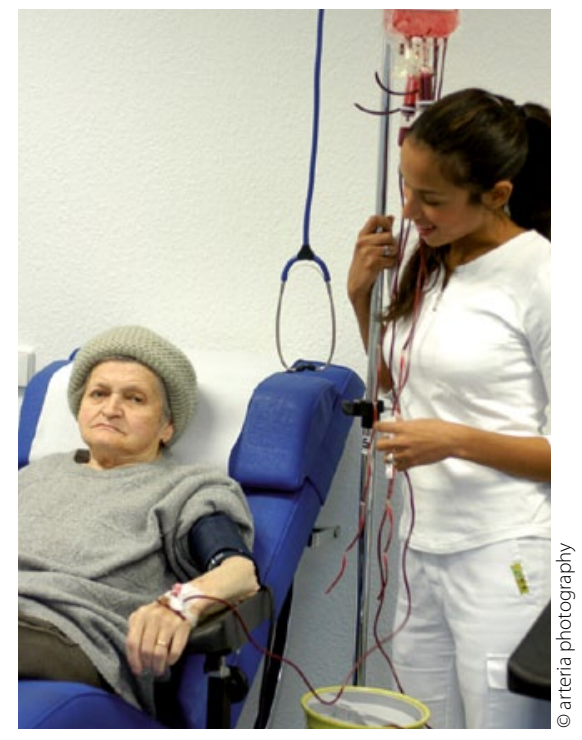

Regelmäßige Transfusionen gehören zum therapeutischen Repertoire bei MDS.

nur 400-500 mg. Daraus ergibt sich die Bedeutung der Chelattherapie.

Rose et al. konnten zeigen, dass Patienten, die eine Chelat-Therapie erhalten, einen deutlichen Überlebensvorteil haben. Indiziert ist diese Behandlung allerdings erst bei einem Ferritinspiegel über $1000 \mathrm{ng} / \mathrm{ml}$ und bei noch ausreichender Lebenserwartung des Patienten. „Ein Hochrisiko-MDS-Patient gehört sicher nicht zu den Kandidaten für eine Eisenchelattherapie“, erklärte Hofmann.

Auch vor einer allogenen Stammzelltransplantation sollten die Patienten mit dieser Behandlung ihre Eisenbilanz verbessern, weil eine Eisenüberladung einen negativen Effekt auf den Verlauf nach der Transplantation haben kann.

\section{Neue Strategien mit guten Ansprechraten}

In einer Studie von Passweg et al., die die Therapie mit Immunsuppressiva mit „Best Supportive Care“ (BSC) verglich, konnte eine Ansprechrate von 30\% und ein klarer Vorteil hinsichtlich des Gesamtüberlebens erreicht werden. Der Nutzen spezifischer Therapien beschränkt sich auf ein gut selektioniertes Patientengut, wie auch eine Studie mit dem Antikörper Alemtuzumab gezeigt hat (Olnes M.J. et al.). Hier erzielte man ein Gesamtansprechen von 83\%; drei Viertel der Patienten wurden unabhängig von Transfusionen.

Lenalidomid hat die Behandlung von MDS-Patienten mit einer singulären Deletion am Chromosom 5 (MDS 5q) revolutioniert. In einer Studie von Fenaux R. et al., bei der Patienten entweder 5 oder $10 \mathrm{mg}$ Lenalidomid oder BSC erhielten, wurden $50-60 \%$ der Patienten unter der höheren Dosierung transfusionsfrei oder erreichten eine volle Remission der Hämatopoese. „Das ist dramatisch gut etwa für Patienten, die seit zehn Jahren alle zwei Wochen zwei EKs bekommen", kommentierte Hofmann.

Im Hinblick auf MDS-Patienten mit einem höheren Risiko wies Hofmann auf die epigenetische Therapie mit 5-Azacytidin hin. Das wichtigste Ergebnis einer randomisierten Studie von Fenaux R. et al. war, dass „die Patienten unter dieser Therapie meist keine komplette Remission erreicht haben, dass sich auch das Blutbild in der Regel nicht dramatisch verbessert hat und sie weiterhin eine supportive Therapie benötigen, sich aber das Überleben signifikant verlängert hat".

MARTIN BISCHOFF *

- Symposium „Akute und chronische Leukämien und maligne Lymphome" anlässlich des 117. Kongresses der DGIM 2011 in Wiesbaden 